Chronique des activités archéologiques de

\title{
Des serpents à Incoronata. La campagne de 2019
}

\section{Mario Denti}

\section{(2) OpenEdition \\ Journals}

\section{Édition électronique}

URL : http://journals.openedition.org/cefr/4562

DOI : $10.4000 /$ cefr.4562

ISSN : 2282-5703

Éditeur

École française de Rome

Référence électronique

Mario Denti, « Des serpents à Incoronata. La campagne de 2019 », Chronique des activités

archéologiques de l'École française de Rome [En ligne], Italie du Sud, mis en ligne le 06 juillet 2020

consulté le 08 juillet 2020. URL : http://journals.openedition.org/cefr/4562 ; DOI : https://doi.org/

$10.4000 /$ cefr. 4562

Ce document a été généré automatiquement le 8 juillet 2020.

(c) École française de Rome 


\title{
Des serpents à Incoronata. La campagne de 2019
}

\author{
Mario Denti
}

\section{NOTE DE L'AUTEUR}

Direction de la fouille : Mario Denti. Assistante : Emmanuelle Smirou (Laboratoire LAHM, UMR 6566 CReAAH, Université Rennes 2). Responsables de secteur : Josipa Mandić, Cesare Vita, Chloé Damay (Laboratoire LAHM, UMR 6566 CReAAH, Université Rennes 2), Francesca Ippolito (University of Groningen). Responsable du laboratoire : Antonia Miola (Università della Basilicata). Responsable de la topographie et de la photogrammétrie : Théo Ben Makhad (Université Rennes 2). Concession :

Soprintendenza Archeologia Belle Arti e Paesaggio della Basilicata, MIBACT DG-ABAP $916 \mathrm{du}$ 30/08/2019. Aide financière : Laboratoire LAHM, Université Rennes 2. Centre Jean Bérard (Naples) - Ministère des Affaires étrangères et du Développement international (Paris). Institutions : Laboratoire LAHM, UMR 6566 CReAAH, Université Rennes 2.

1 Le programme de la dix-septième campagne de fouille à Incoronata, qui a eu lieu au mois de septembre 2019, a vu la poursuite de l'exploration des contextes précédemment mis au jour dans le secteur sud-occidental de la colline (fig. 1), avec un double objectif : à travers la fouille en profondeur, la connaissance des phases les plus anciennes de structures, espaces et bâtiments dont nous saisissons les niveaux d'utilisation les plus récents; à travers l'élargissement de la surface de fouille, une meilleure compréhension de la morphologie de structures, espaces et bâtiments dont l'extension nous est actuellement connue seulement en partie. Les résultats de la campagne ont été particulièrement importants. 


\section{Le secteur Sud : des nouvelles structures bâties}

Dans le secteur Sud de la fouille (fig. 1) nous avons élargi l'exploration de l'espace entourant vers le sud le contexte cultuel chtonien datable des IX $^{\mathrm{e}}$-VIII ${ }^{\mathrm{e}}$ siècles $^{1}$, pour essayer de l'inscrire davantage dans son environnement fonctionnel. Comme nous l'avons vu l'année précédente ${ }^{2}$, la principale découverte de ce secteur est représentée par un enclos de pierres en forme de triangle isoscèle, parfaitement orienté est-ouest (fig. 2, A), érigé sur le plan d'oblitération d'une grande fosse de forme subcirculaire creusée dans le sol géologique (fig. 2, B). Celle-ci avait été progressivement remplie par la superposition de fines strates de terre noircie contenant des ossements animaux carbonisés et de la céramique, évident résultat d'une séquence d'actions rituelles. Exactement à l'angle oriental de cet enclos, aménagé de manière particulièrement soignée, se trouve une grande pierre, interprétable soit comme un autel soit comme un rocher cultuel aniconique (fig. 2, C). Tout autour d'elle, des pratiques rituelles, comprenant des libations et des repas, avaient été réalisées, comme en témoigne la déposition intentionnelle de gros galets, de céramique cérémonielle indigène (figuline et à impasto) ou de nombreux ossements d'animaux. Nous avons eu également l'occasion de souligner l'importance cruciale de cette découverte, qui s'inscrit directement dans une typologie architecturale attestée dans le milieu égéen de l'âge du Fer, où nous la trouvons constamment associée à des contextes rituels propres aux cultes des ancêtres, voire des héros. La destination chtonienne de cet ensemble cultuel nous avait d'ailleurs déjà été suggérée par la modalité de l'aménagement de ces structures (toutes hypogées), par les complexes modalités rituelles de leur oblitération et par la nature des objets déposés.

Fig. 1 - Planimétrie de la fouille (DAO T. Ben Makhad).

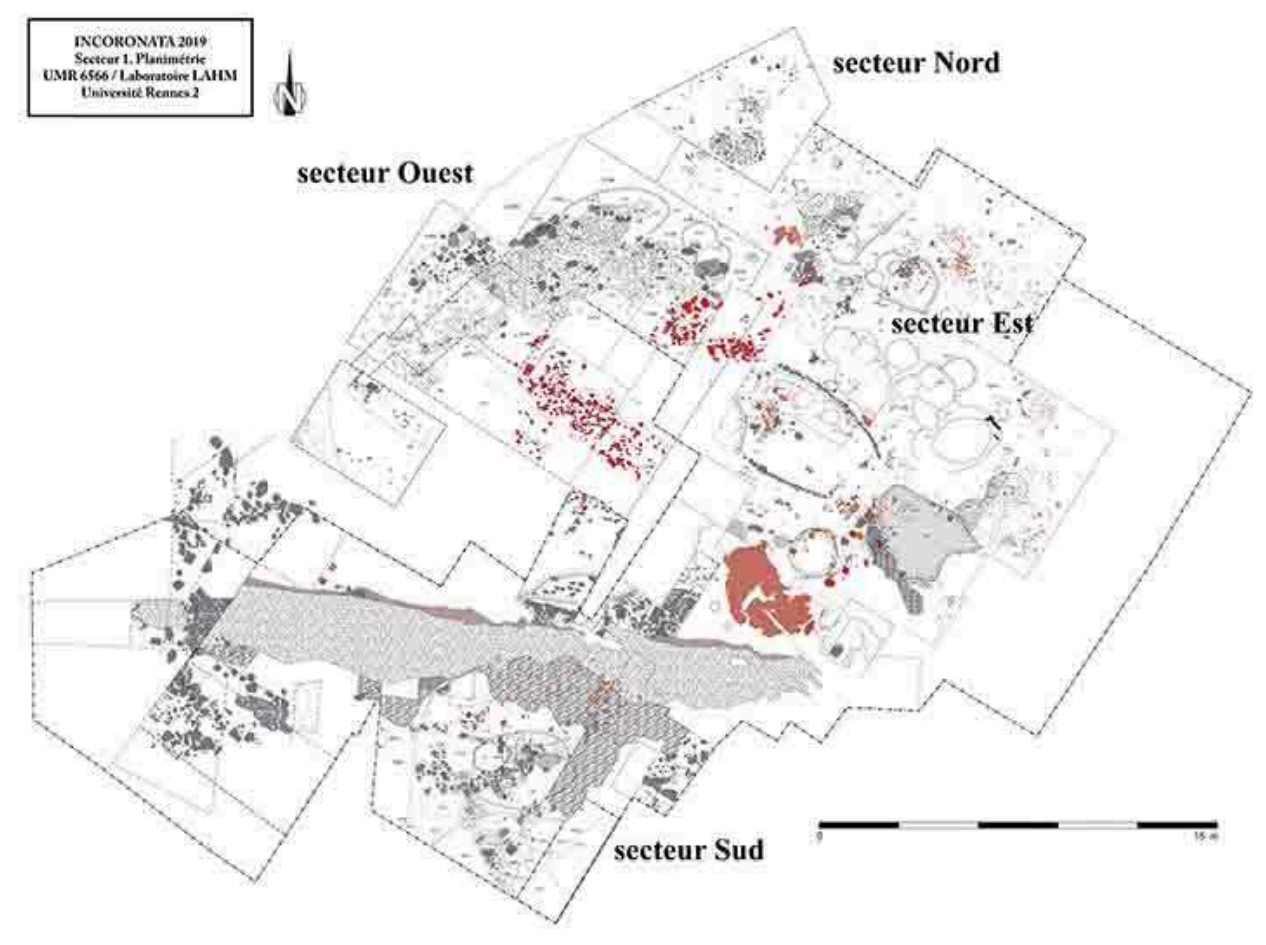


Fig. 2 - Photogrammétrie du secteur Sud (élaboration T. Ben Makhad).

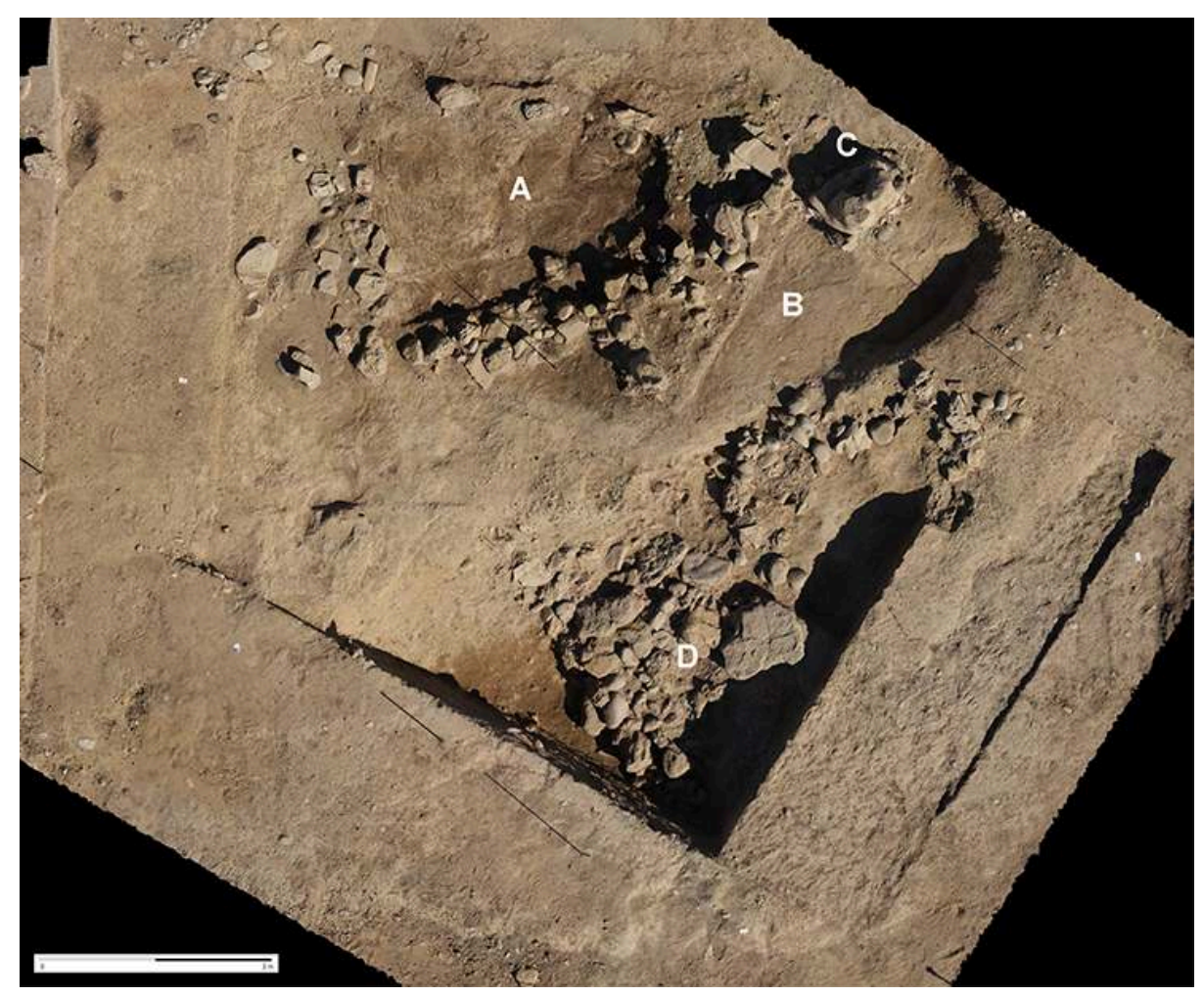

3 La centralité et le rôle éminent de cet espace au sein de la plus ancienne phase d'occupation de la colline actuellement connue ont été confirmés par l'élargissement de la fouille au sud en 2019. Nous y avons intercepté une imposante structure, appuyée sur l'important dénivelé morphologique de la colline qui descend progressivement en altitude vers le sud, composée de gros blocs de pierres et orientée nord-sud (fig. 2, D ; fig. 3). Mesurant pour la partie actuellement mise au jour 3,10 $\times 2 \mathrm{~m}$, elle se développe sur trois niveaux de pierres, qui s'appuient sur un espace artificiellement excavé dans le sol géologique. En l'état actuel de nos connaissances, la fonction de cet aménagement nous échappe. La frappante monumentalité de sa réalisation confirme en tout cas le fait que l'enclos triangulaire ne devait pas, bien évidemment, surgir isolé, aussi bien que tout l'intérêt qui avait été réservé à cet endroit. Peut-être s'agit-il (pure hypothèse de travail) d'une volée de marches constituant l'accès, depuis la vallée, à cet espace cultuel? 
Fig. 3 - Secteur Sud : le contexte cultuel chtonien du IXe-VIII' siècle, vu de l'ouest, avec l'enclos triangulaire, la pierre aniconique et, vers le sud, la partie de la structure en pierres mise au jour (cliché M. Denti).

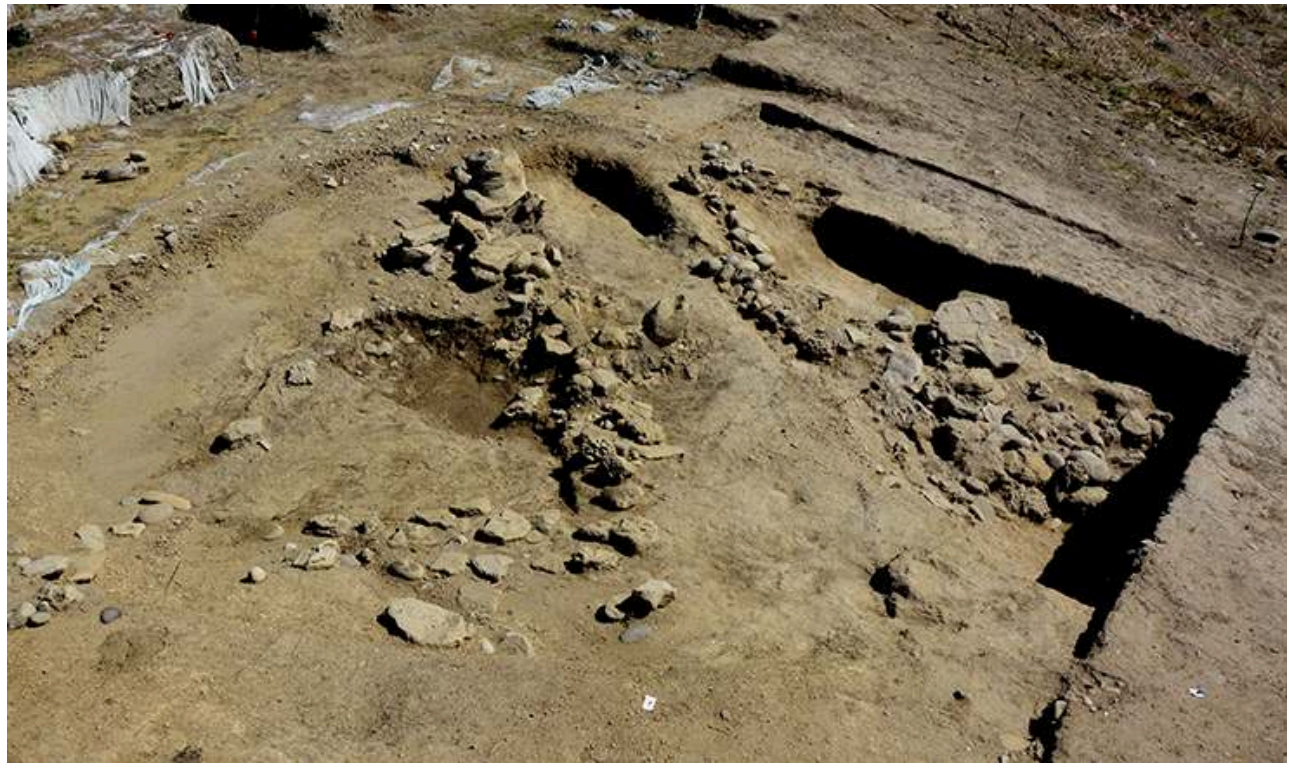

\section{Le secteur Est : des fosses et des serpents}

Plus au nord, dans le secteur où les structures actuellement connues datent du VII ${ }^{e}$ siècle, nous avons poursuivi l'exploration de l'espace qui s'étend au nord-est de l'édifice absidé (fig. 1), en entamant en même temps la fouille à l'intérieur de ce dernier (fig. 4 ; fig. 5, A). Ici, nous avons procédé à l'enlèvement du côté septentrional de la couche sur laquelle gisait la déposition du contexte rituel se trouvant au milieu de l'abside, correspondant à son dernier niveau de fréquentation et datable à l'intérieur de la seconde moitié du VII ${ }^{e}$ siècle ${ }^{3}$. Alignées tout au long du périmètre de l'édifice, sont apparues trois fosses peu profondes, de forme parfaitement circulaire, mesurant $80 \mathrm{~cm}$ de diamètre moyen et 15-20 cm en profondeur (fig. 4 ; fig. 5, B, C, D). Elles avaient été remplies de terre, foncée ou claire, contenant de petits ossements d'animaux et du charbon de bois. Parmi les os, ont été identifiés une fusaïole coupée en deux, des fragments de grands conteneurs, de la céramique indigène achrome et monochrome, de la céramique à impasto; dans deux de ces fosses ont également été déposés des fragments de skyphoi de production locale. Le matériel de ces remplissages permet de les dater toujours au sein du $\mathrm{VII}^{\mathrm{e}}$ siècle, tandis que la morphologie des fosses suggère qu'elles pourraient avoir été destinées à recevoir l'installation de grands conteneurs : une circonstance très cohérente avec la vocation résidentielle-rituelle de ce bâtiment. 
Fig. 4 - Secteur Est : les trois fosses circulaires retrouvées à l'intérieur du côté septentrional de l'édifice absidé, vues de l'ouest (cliché M. Denti).

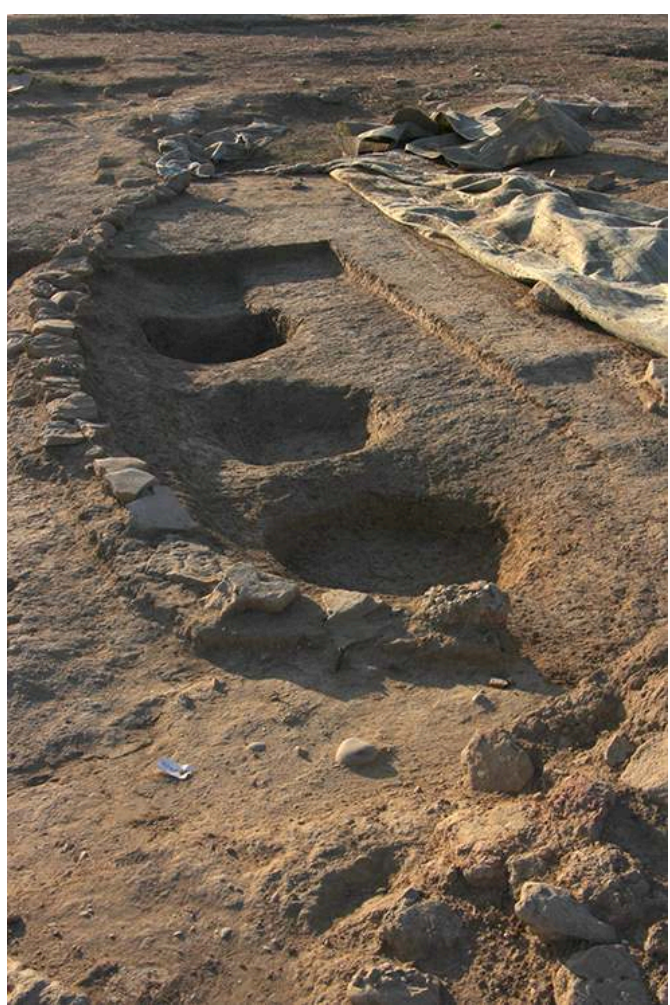

Fig. 5 - Photogrammétrie du secteur Est (élaboration T. Ben Makhad).

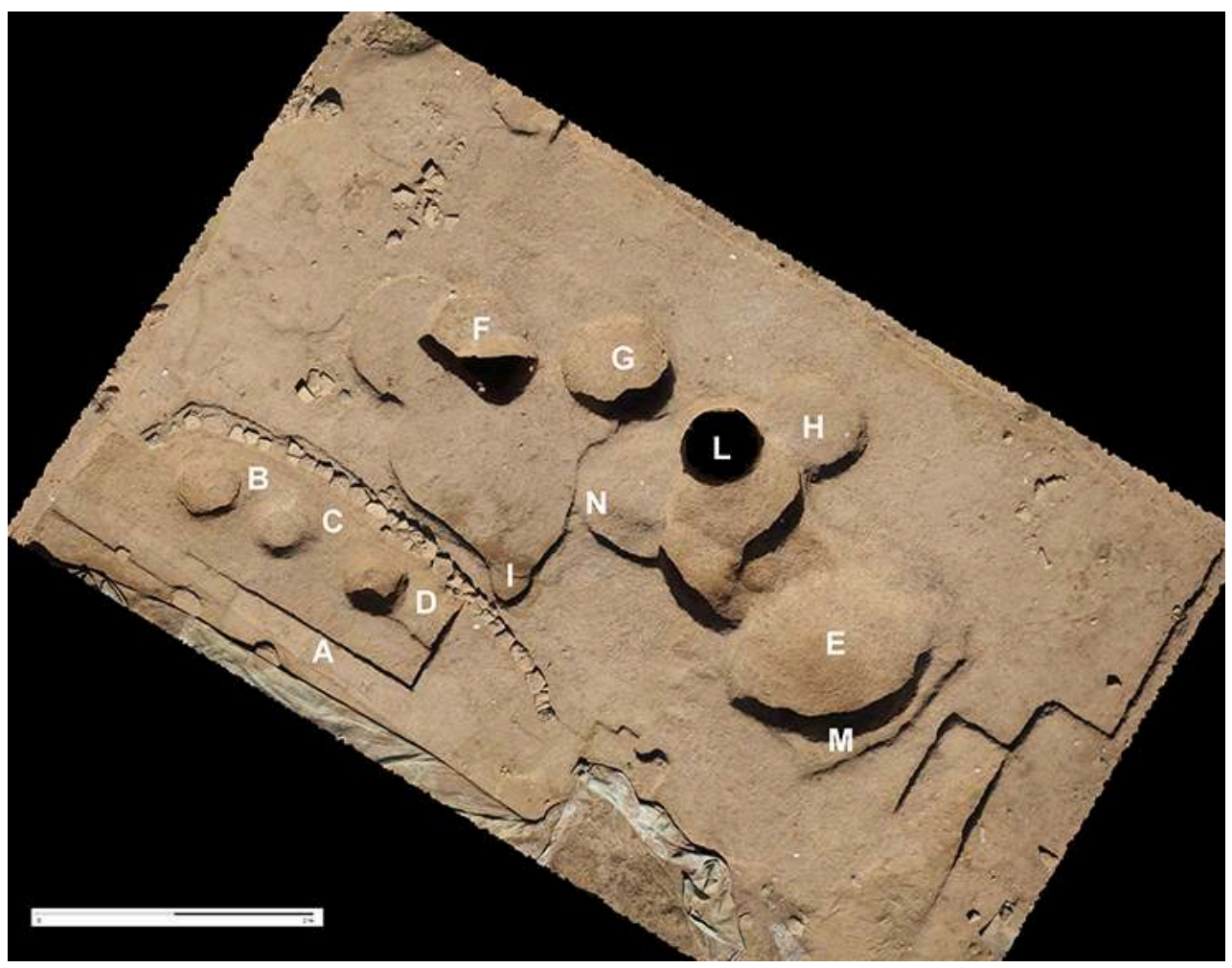

5 À l'extérieur de l'édifice nous avons procédé à l'enlèvement de la couche connectée à l'utilisation de l'espace contenant les deux petits fours pour la torréfaction de glands, 
mis au jour en 2018 dans un état de conservation exceptionnel et associés à des dizaines de glands de chêne et aux restes de leurs récipients (des conteneurs à impasto, achromes ou monochromes) aussi bien qu'à d'importants restes de bois carbonisét. Cet espace, qui s'ouvre en correspondance de l'angle oriental de l'entrée de l'édifice absidé (fig. 5, E), s'est révélé - comme pour la plupart des structures fouillées sur la colline directement creusé dans le sol géologique. Il forme une cavité ovale peu profonde (diamètre max. 4,10 m, profondeur entre 20 et $40 \mathrm{~cm}$ ), caractérisé par la présence, sur son côté sud-est, d'un deuxième bord rectiligne formant un gradin (une sorte de banquette) (fig. 5, M).

6 Au nord de cet espace, une série de trois fosses de forme parfaitement circulaire, orientées en sens est-ouest, est apparue (fig. 5, F, G, H). La partie supérieure de leur oblitération était marquée par la déposition, à la verticale, d'éléments en bronze et en fer. Cette oblitération - dont les remplissages n'ont pas été encore fouillés - correspond stratigraphiquement à l'aménagement, juste à côté de la paroi externe de l'édifice absidé (fig. 5, I), d'une déposition rituelle comprenant un pithos associé à une petite olla monochrome intentionnellement fragmentée in situ; cette dernière a pu être entièrement reconstituée (fig. 6), tandis que le pithos ne l'a été que pour sa partie inférieure, jusqu'à $40 \mathrm{~cm}$ de hauteur environ.

Fig. 6 - Secteur Est : la petite olla monochrome déposée et fragmentée in situ à l'extérieur de l'édifice absidé (cliché M. Denti).

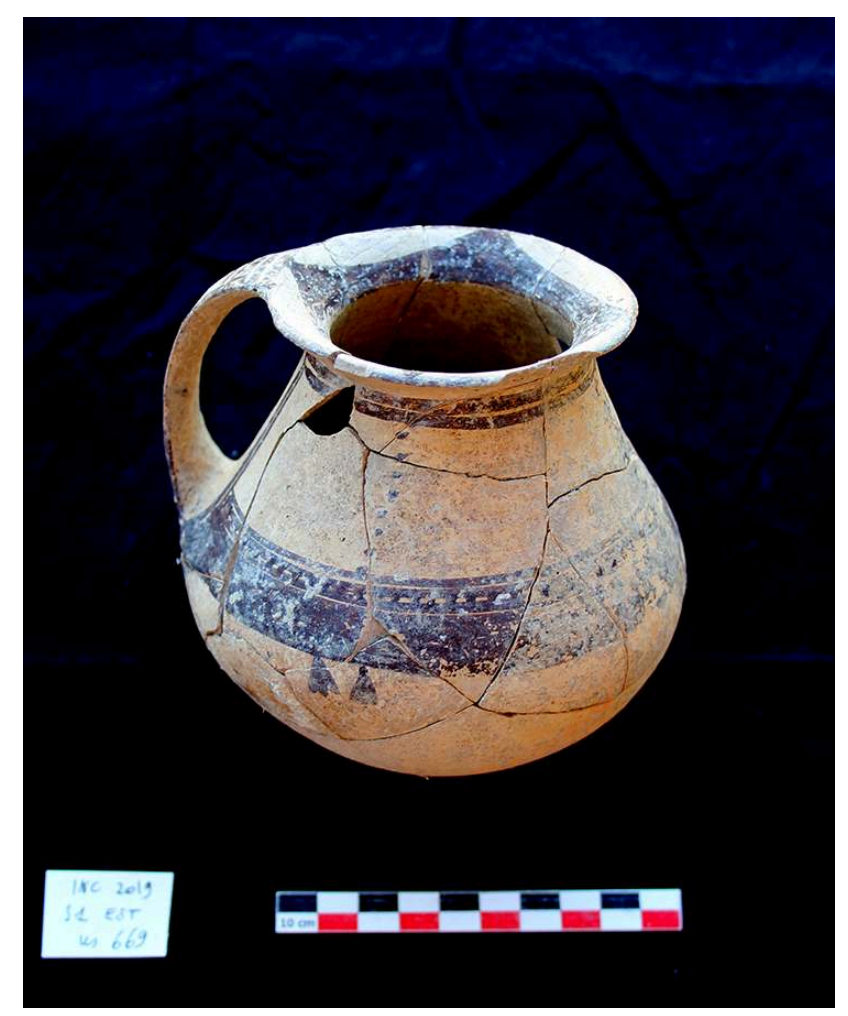

Entre la fosse $\mathrm{G}$ et $\mathrm{H}$ nous avons mis au jour une autre fosse circulaire, laquelle se signale pour sa morphologie particulière, inscrite dans une typologie que nous n'avions pas encore rencontrée à Incoronata (fig. 5, L). De forme parfaitement cylindrique (diamètre : $1,05 \mathrm{~m}$ ), elle est profonde d'au moins 2 mètres (profondeur explorée par la fouille, qui n'est pas achevée) et elle présente, à $1,80 \mathrm{~m}$ de profondeur, un élargissement du profil de la paroi vers l'extérieur, sur une distance que nous n'avons 
pu encore établir, donnant à l'ensemble un profil piriforme. Cette fosse est entourée au sud par une série de dépressions descendant progressivement vers elle, dotées d'un rebord bombé, mesurant $30-40 \mathrm{~cm}$, qui se développent tout au long de ses limites (fig. 5, N).

8 Une autre caractéristique majeure de cette fosse cylindrique est représentée par la modalité et le contenu de son oblitération (US 747 et 762), qui permettent d'en saisir au moins en relation au moment de son utilisation dernière - la couleur rituelle. La terre du comblement contenait en effet des ossements, des galets ayant subi l'action du feu, des coquillages, associés à de nombreux tessons de céramique, appartenant à de grands conteneurs, à de la céramique achrome, monochrome, à impasto; une fusaïole fragmentée et une bobine; deux kotylai et un krateriskos PC d'importation (fig. 7) et les parois d'un stamnos peint de production locale (fig. 8). Deux grandes pierres aniconiques avaient été intentionnellement fixées à la verticale à l'intérieur de ce comblement, en correspondance d'une déposition à plat de galets, petites pierres et céramiques (fig. 9). Les deux pierres avaient été soigneusement choisies par leur forme, aspect et dimensions (fig. 10): une, plus grande (h. $45 \mathrm{~cm}, 1.28 \mathrm{~cm}$ ), présente une surface très lisse ; l'autre, plus petite $(\mathrm{h} .31 \mathrm{~cm}, 1.14 \mathrm{~cm})$, rugueuse en surface, avait été retravaillée pour lui donner une morphologie, avec la partie inférieure en pointe, capable de la fixer à la verticale dans la terre.

Fig. 7 - Secteur Est : céramique PC d'importation, dans la couche d'oblitération de la fosse cylindrique (cliché M. Denti).

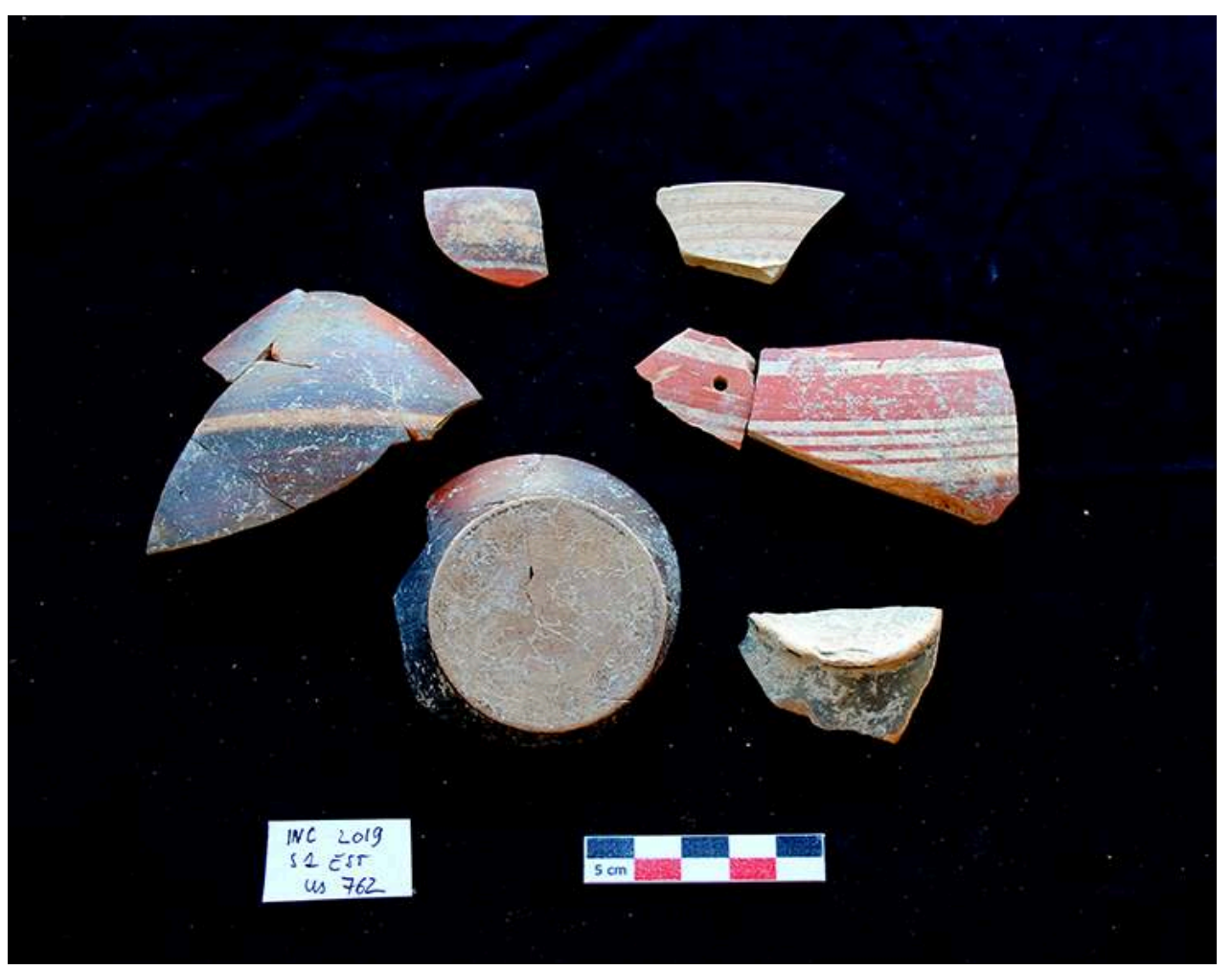


Fig. 8 - Secteur Est : parois et anse du stamnos de production locale, dans la couche d'oblitération de la fosse cylindrique (cliché M. Denti).

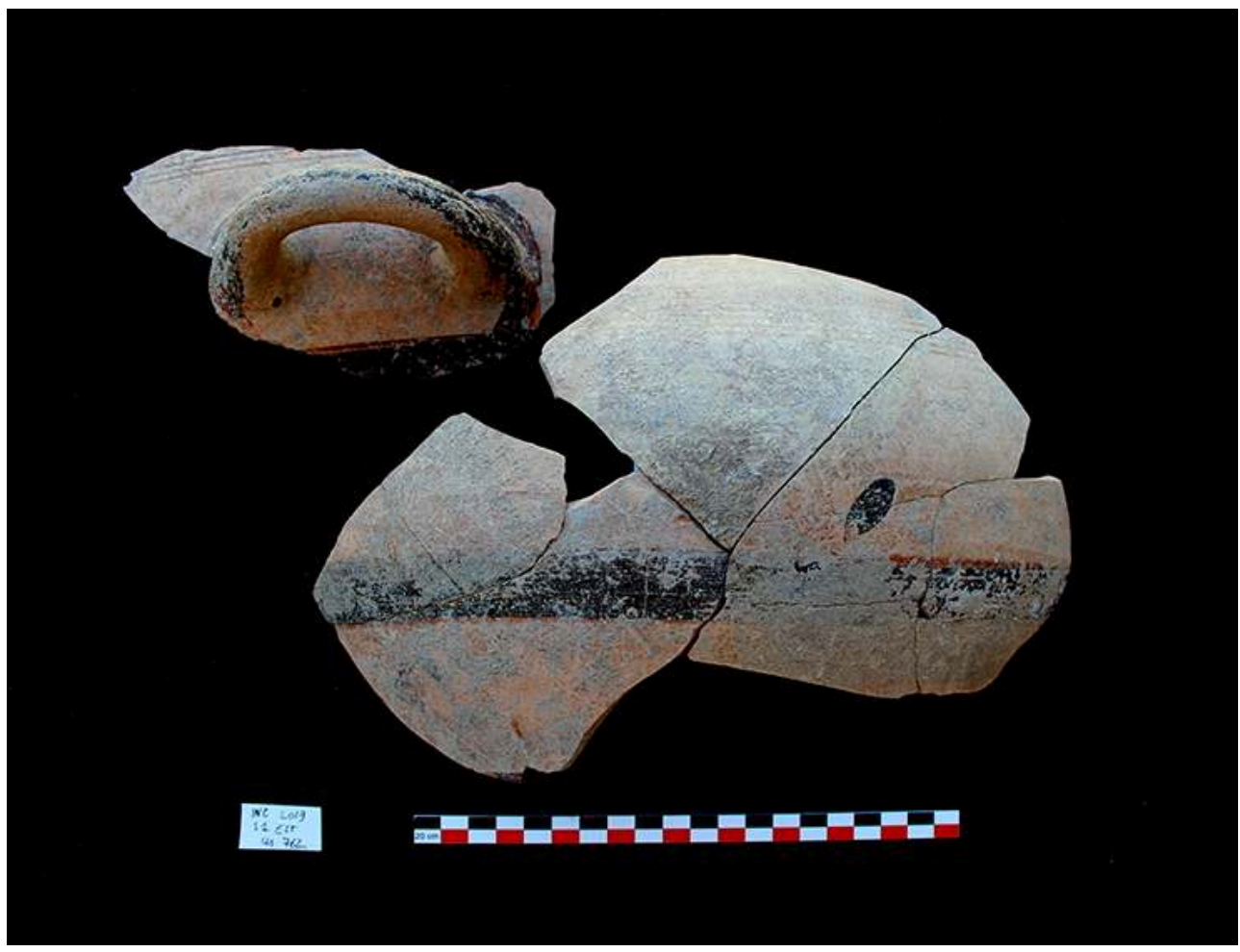

Fig. 9 - Secteur Est : le niveau intermédiaire de l'oblitération de la fosse cylindrique, avec la déposition de galets, petites pierres, céramiques et de deux grandes pierres fixées à la verticale (cliché M. Denti).

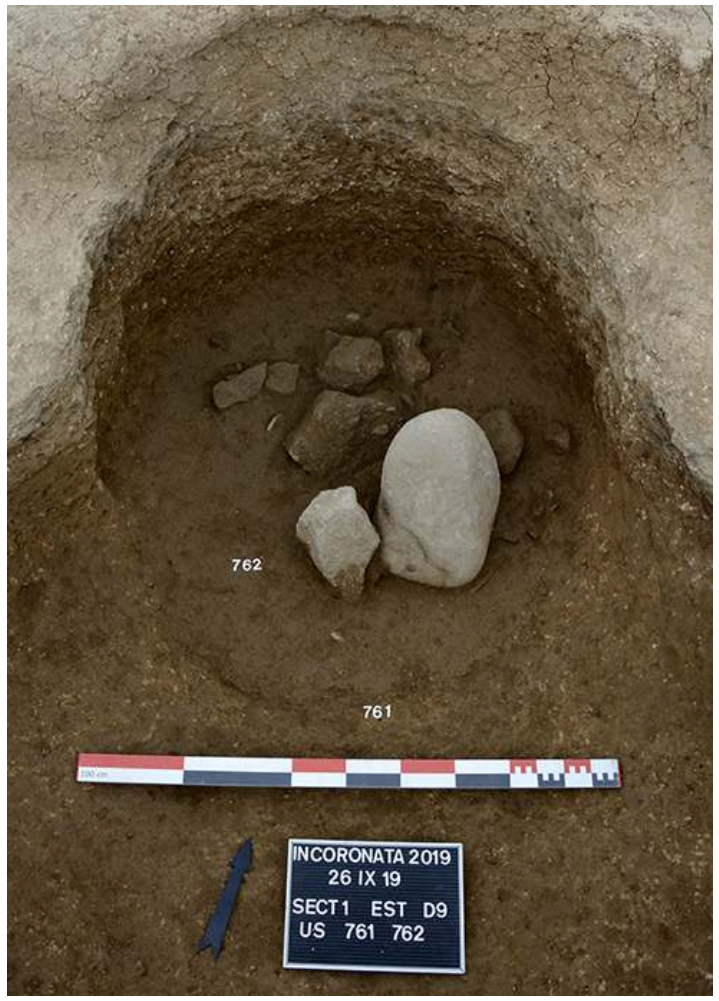


Fig. 10 - Secteur Est : les deux pierres provenant de l'oblitération de la fosse cylindrique (cliché M. Denti).

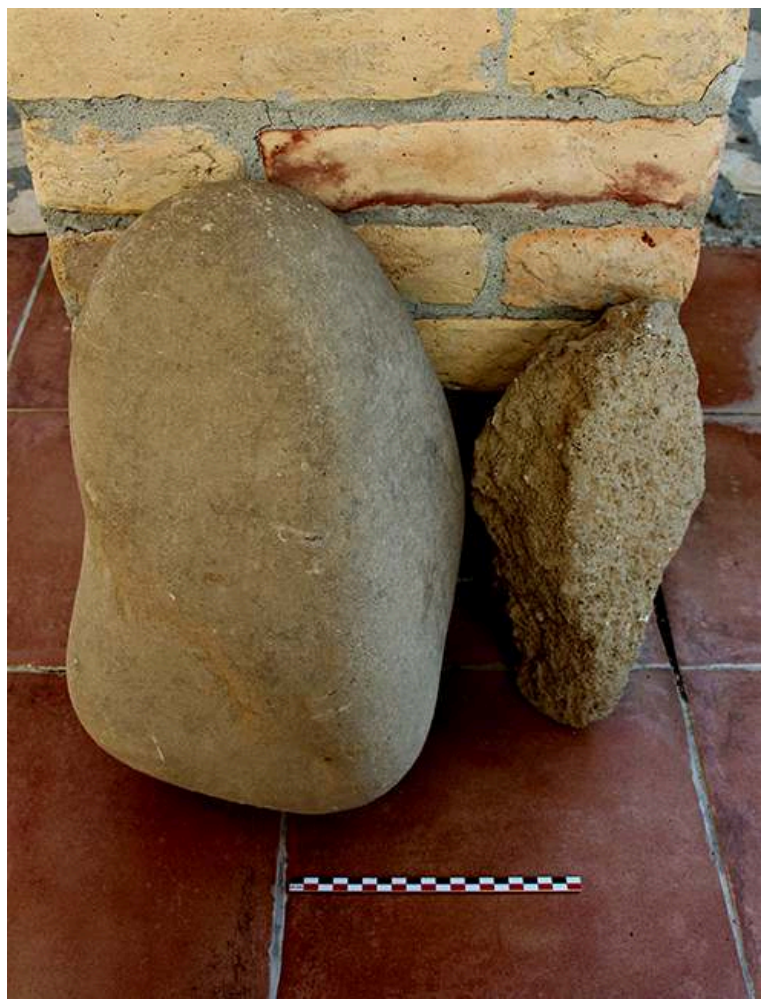

Particulièrement remarquable a été la découverte, dans le niveau supérieur de cette couche de remplissage (US 347) caractérisée par la présence de nombreux os noircis et des charbons de bois, à $20 \mathrm{~cm}$ du bord de la fosse (fig. 18, B2), d'un fragment d'une petite sculpture en terre cuite, représentant un serpent, dont seule la tête est conservée (fig. 11). L'argile, de couleur orange rosé (typique de la céramique de production locale) a été peinte en vernis brunâtre, très foncé, sur la seule partie supérieure du corps de l'animal. La tête, qui mesure $2,5 \times 0,5 \times 3 \mathrm{~cm}$, présente la bouche ouverte, les yeux incisés en forme de triangle et est réalisée avec une grande finesse naturaliste. La déposition de la représentation d'un serpent ne peut que confirmer le caractère profondément chtonien de ce contexte. 
Fig. 11 - Secteur Est : la tête du petit serpent en terre cuite, provenant du comblement de la fosse cylindrique (cliché M. Denti).

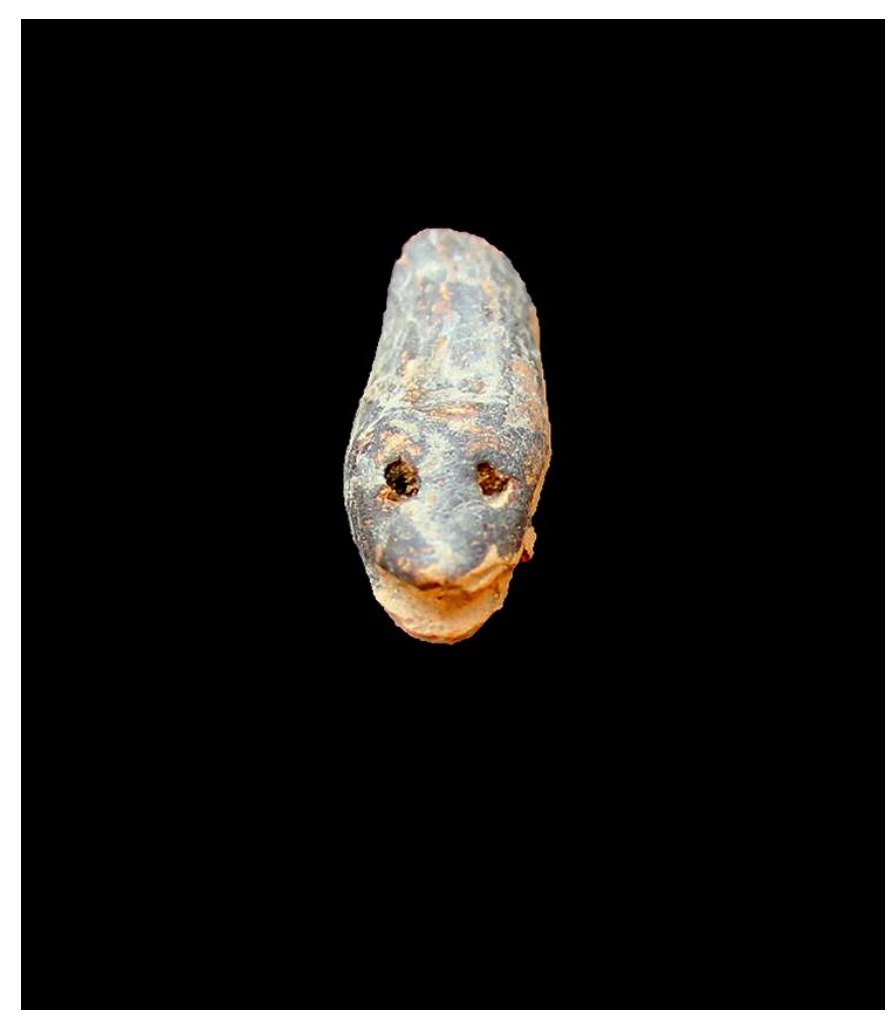

10 Nous avons pu également observer une circonstance du plus grand intérêt pour la compréhension du fonctionnement de l'ensemble de l'espace rituel entourant au nord l'édifice absidé. À cette même sculpture de serpent appartient en effet un autre segment, relatif au corps de l'animal, serpentant autour d'un élément perdu (fig. 12), que nous avions découvert en 2018 à quelques mètres plus au nord (US 527), déposé sur un lit de galets encadrant un bothros, associé à des skyphoi et d'ossements d'animaux (cf. fig. 18, B1 $)^{5}$. Cette extraordinaire correspondance témoigne au moins de trois situations : du caractère unitaire des actions rituelles pratiquées, dans le temps comme dans l'espace, dans cette zone de l'espace cultuel d'Incoronata (un aspect sur lequel nous devrions revenir par la suite); de la couleur uniformément chtonienne de ces cérémonies, en confirmant les conclusions auxquelles nous étions auparavant parvenus sur la base de la typologie des actions rituelles ; de la pratique rituelle de l'enchainment ${ }^{6}$, que nous avons pu amplement observer dans d'autres circonstances à Incoronata ${ }^{7}$. 
Fig. 12 - Incoronata 2019 et 2018 : la tête e le segment du corps du serpent en terre cuite, provenant de deux contextes différents ; le premier déposé dans I'US 347 , le deuxième dans I'US 527 (cliché M. Denti).

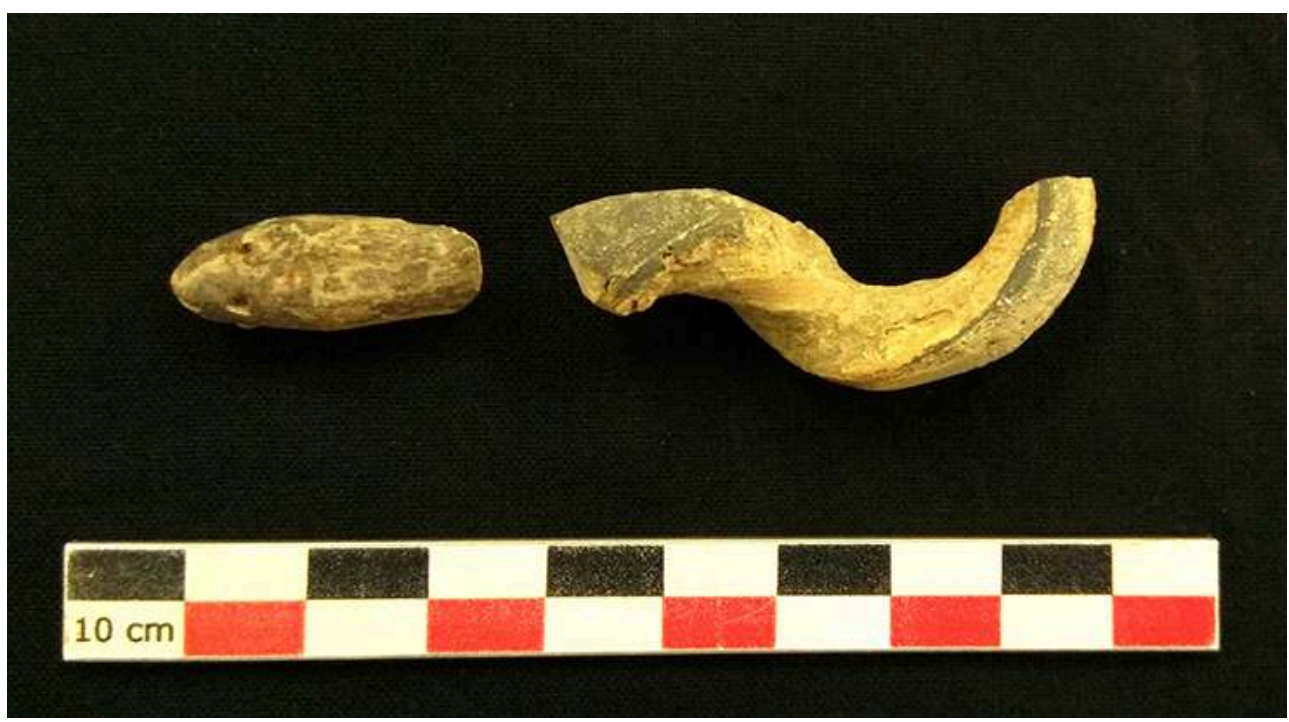

En revenant au petit serpent, nous pourrions imaginer qu'il ait pu appartenir, pour ce niveau chronologique, à une sculpture de grandes dimensions : comme un élément de l'égide d'une image d'Athéna, ou comme l'attribut d'une représentation de la Gorgone Méduse, ou encore d'une autre divinité féminine de type chtonien ; il aurait également pu faire partie de la décoration plastique d'un exemplaire de vaisselle rituelle, par exemple les serpents plastiques qui décorent les céramiques protoattiques contemporaines du Céramique d'Athènes.

\section{Le secteur Nord : de multiples dépositions rituelles}

Dans la limite septentrionale de la zone de fouille (fig. 1, 18), nous avons entamé l'exploration d'un contexte rituel qui apparaît complètement recouvert par une grande accumulation de gros galets, uniformes par leur taille, leur couleur et leur conformation (fig. 13). Cette concentration de galets, insérés dans un tas de terre très compacte de couleur marron foncé, est organisée sur quatre niveaux, et contenait à l'intérieur des os et de petits tessons de céramique très usés: indigène (dont de nombreuses parois appartenant à une olla bichrome gisant à plat), grecque locale datable du VII ${ }^{\mathrm{e}}$ siècle, des anses d'amphores SOS et corinthiennes (ces derniers avec des incisions). Cette accumulation affichait des caractéristiques particulières: elle ne contenait aucun matériel de construction (comme c'est le cas de la plupart des recouvrements limitrophes) et la terre avait été choisie d'une coloration précise, un marron spécialement foncé, qui la distinguait de tous les autres tas d'oblitération que nous avons fouillés dans ce secteur. Il s'agit donc d'une couverture " éminente ", isolée, destinée sans doute à protéger quelque chose d'important. 
Fig. 13 - Secteur Nord : la partie supérieure de l'accumulation des gros galets, vue du nord (cliché M. Denti).

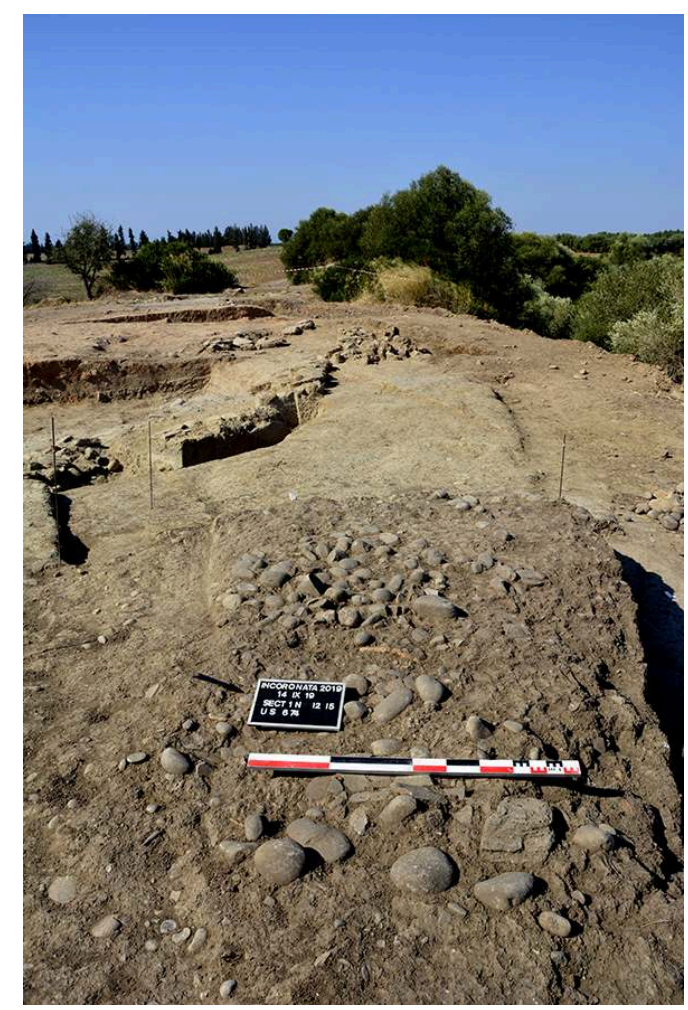

13 Ce n'est pas donc un hasard si, une fois retiré ce recouvrement, il est apparu un aménagement rituel particulièrement remarquable. Celui-ci est constitué par un lit de galets de taille moyenne/petite, sur lequel gisaient des restes de repas et de libations (fig. 14 ; fig. 18, A1) : des ossements ; le crâne d'un veau de quelques mois d'âge, dont la partie antérieure - découpée verticalement - avait été retirée, déposée à plat sur la nuque et dont des parties de la mâchoire et des dents gisaient tout autour (fig. 15), au voisinage de tessons appartenant à des skyphoi du PC Moyen et Tardif d'importation. Cet aménagement encadrait du côté nord une structure concave, avec un profil ovale/ circulaire, scellée par des galets posés sur un lit d'écailles de pierres et de briques (fig. 14 ; fig. 18, C). Nous avons seulement commencé à libérer cette structure des petits galets et de la terre qui en recouvraient le niveau supérieur, lequel est apparu comme caractérisé (fig. 16) par une couche très compacte de gravillon, de microfragments de briques, de petits cailloux et de pierres. Celles-ci avaient été placées en particulier tout au long des bords, tandis que certaines, à la verticale, semblent descendre en profondeur. Depuis les bords, vers le centre, se configure une dépression (actuellement de $15-20 \mathrm{~cm}$ de profondeur) au centre de laquelle un grand galet de forme ovoïde a été intentionnellement déposé (fig. 16). 
Fig. 14 - Secteur Nord : le lit de galets, avec les restes de repas et de libations, entourant au nord la structure ovale/circulaire concave, dont le niveau supérieur a été scellé par des petits galets (US 738) (cliché M. Denti).

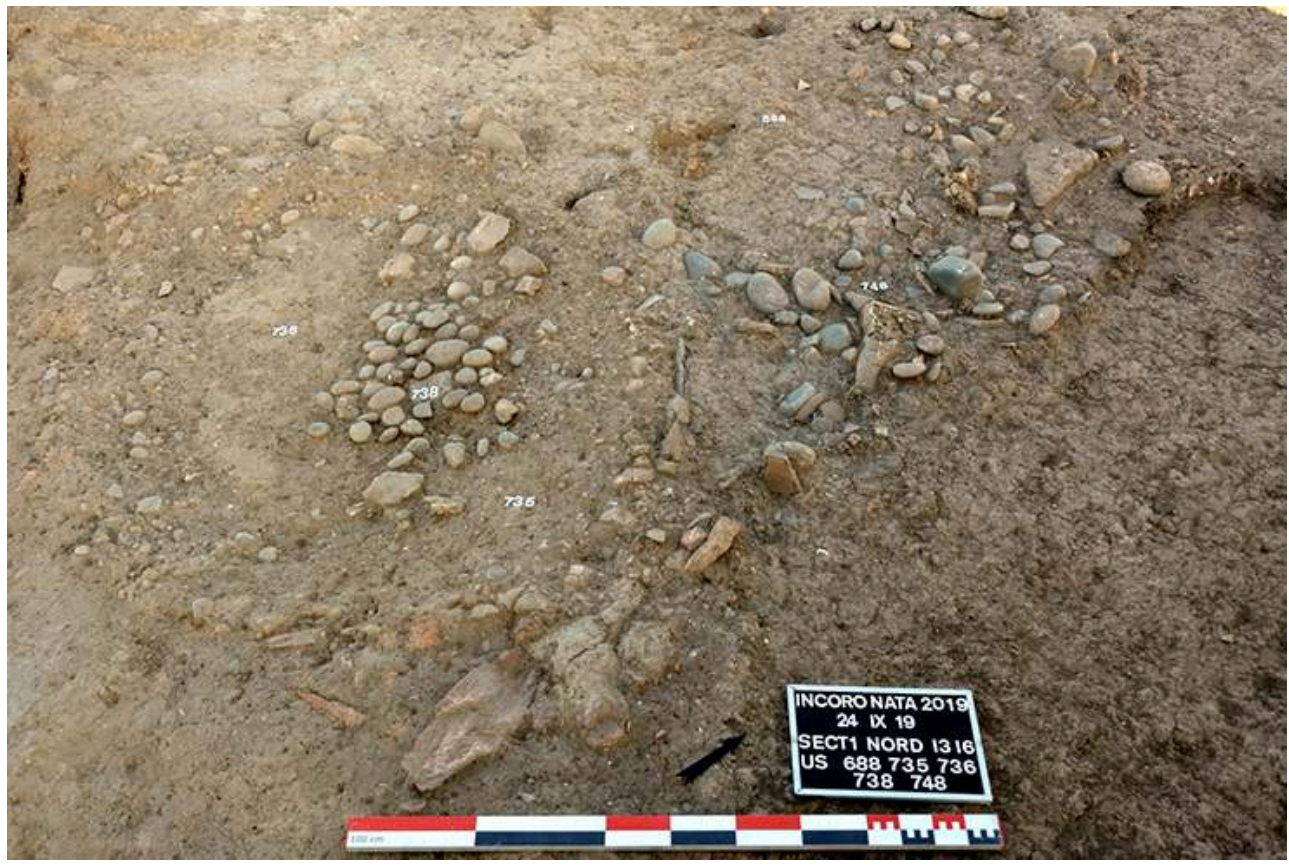

Fig. 15 - Secteur Nord : le crâne du veau, découpé verticalement et déposé à plat sur le lit de galets (cliché M. Denti).

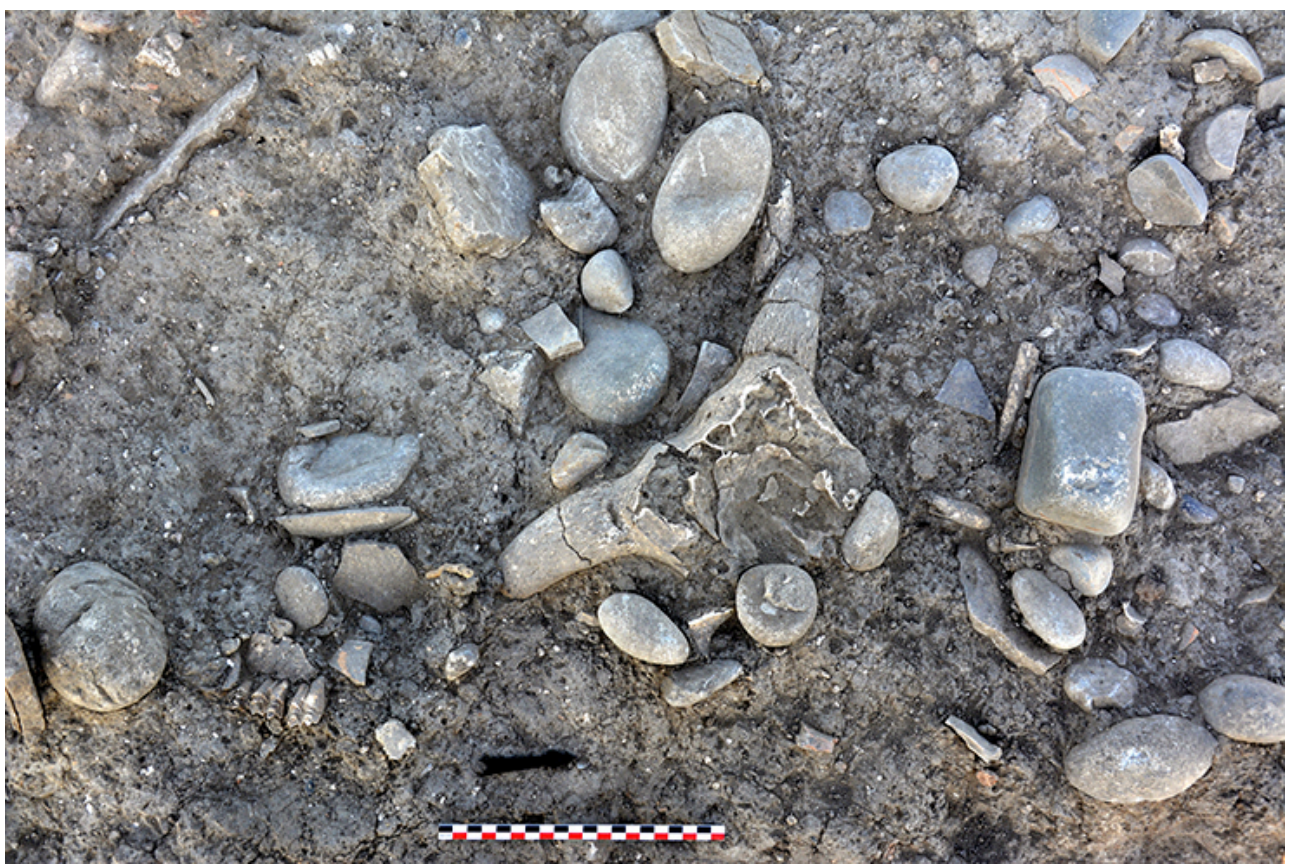


Fig. 16 - Secteur Nord : la structure ovale/circulaire concave, après l'enlèvement de la couche supérieure de couverture, avec le grand galet de forme ovoïde posé au centre (cliché M. Denti).

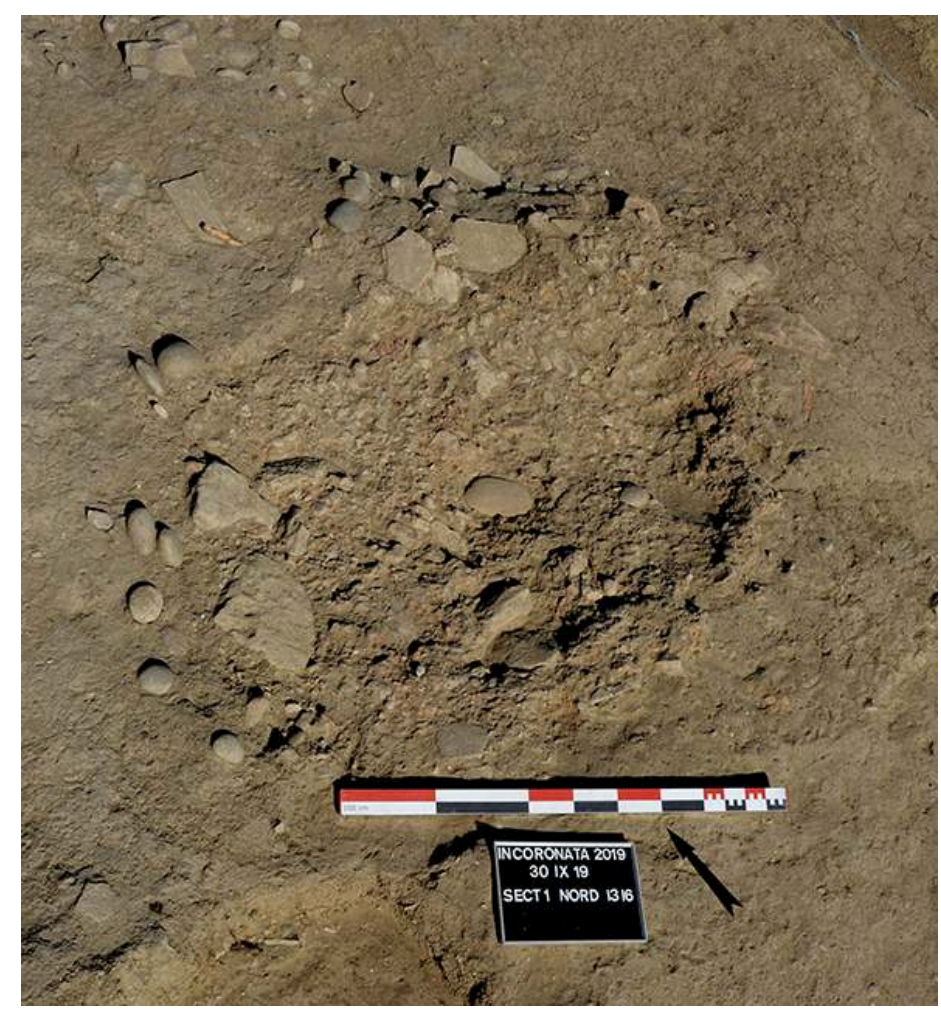

L'usage de placer au centre d'un espace cérémoniel défini un grand galet, émergeant par sa forme, ses dimensions et sa couleur, est attesté dans des contextes rituels à caractère funéraire dans le milieu cycladique, comme le montrent les exemples récemment étudiés par Nota Kourou à Tenos, datant du Géométrique jusqu'au V $V^{e}$ siècle ${ }^{8}$. La proximité d'Incoronata avec le monde cycladique est d'ailleurs un élément constant, que nous sommes aujourd'hui en mesure d'observer de manière croissante dans plusieurs domaines : architecture, céramique, pratiques rituelles.

Sur la base de la comparaison avec un aménagement analogue que nous avons mis au jour à quelque mètre à l'est (lit de galets entourant un bothros), il pourrait peut-être s'agir du «bouchon » d'une fosse, même si cette morphologie spécifique du scellement (avec des gravillons le rendant très compact, et un grand galet au centre) apparaît pour la première fois à Incoronata. Le contexte adjacent comprenait en effet un ample aménagement de galets réguliers et de petite taille (US 527, fig. 18, A2, B1), posés à plat directement sur la terre sur plusieurs niveaux, entourant un bothros associé à un autel de pierres (fig. 18, D). Sur ce véritable tapis gisait une remarquable quantité d'ossements d'animaux et de tessons appartenant pour la plupart à des skyphoi de production locale, témoignant de la réalisation de pratiques rituelles impliquant la consommation de viande et de vin ${ }^{10}$.

La continuité et l'homogénéité topographiques, stratigraphiques, fonctionnelles et chronologiques de ces deux contextes nous indiquent que nous nous trouvons dans un même espace rituel, dans lequel des actions cérémonielles ont été réalisées pendant la deuxième moitié du VII siècle. Cette homogénéité est suggérée non seulement par l'identité morphologique de l'aménagement (comprenant des bothroi contournés au nord par des lits de galets sur lesquels gisent les restes des actions sacrificielles et 
libatoires) mais également par la déposition, dans les deux contextes, de fragments de céramique appartenant à un même vase. Il s'agit d'une œnochoé figurée de fabrication locale, une production caractérisée par un "plurilinguisme figuratif » typique ${ }^{11}$ qui mélange des styles et des répertoires différenciés (fig. 17) : un schéma décoratif de type cycladique sur le col (double volute horizontale); des motifs gréco-orientaux, comme des éléments serpentiformes pointillés et, surtout, une chèvre sauvage WGS sur l'épaule; des grandes volutes terminant en crochet sur la panse. L'état de conservation est différencié entre les fragments appartenant au col, qui ont subi des altérations de température au contact avec le feu, et les fragments de la panse, qui ont mieux préservé leur surface originelle ${ }^{12}$.

Fig. 17 - Secteur Nord et Ouest : les fragments de l'œnochoé figurée de production locale (noter sur le haut de l'épaule la tête de la chèvre sauvage), déposés dans trois contextes différents (cliché J. Mandić).

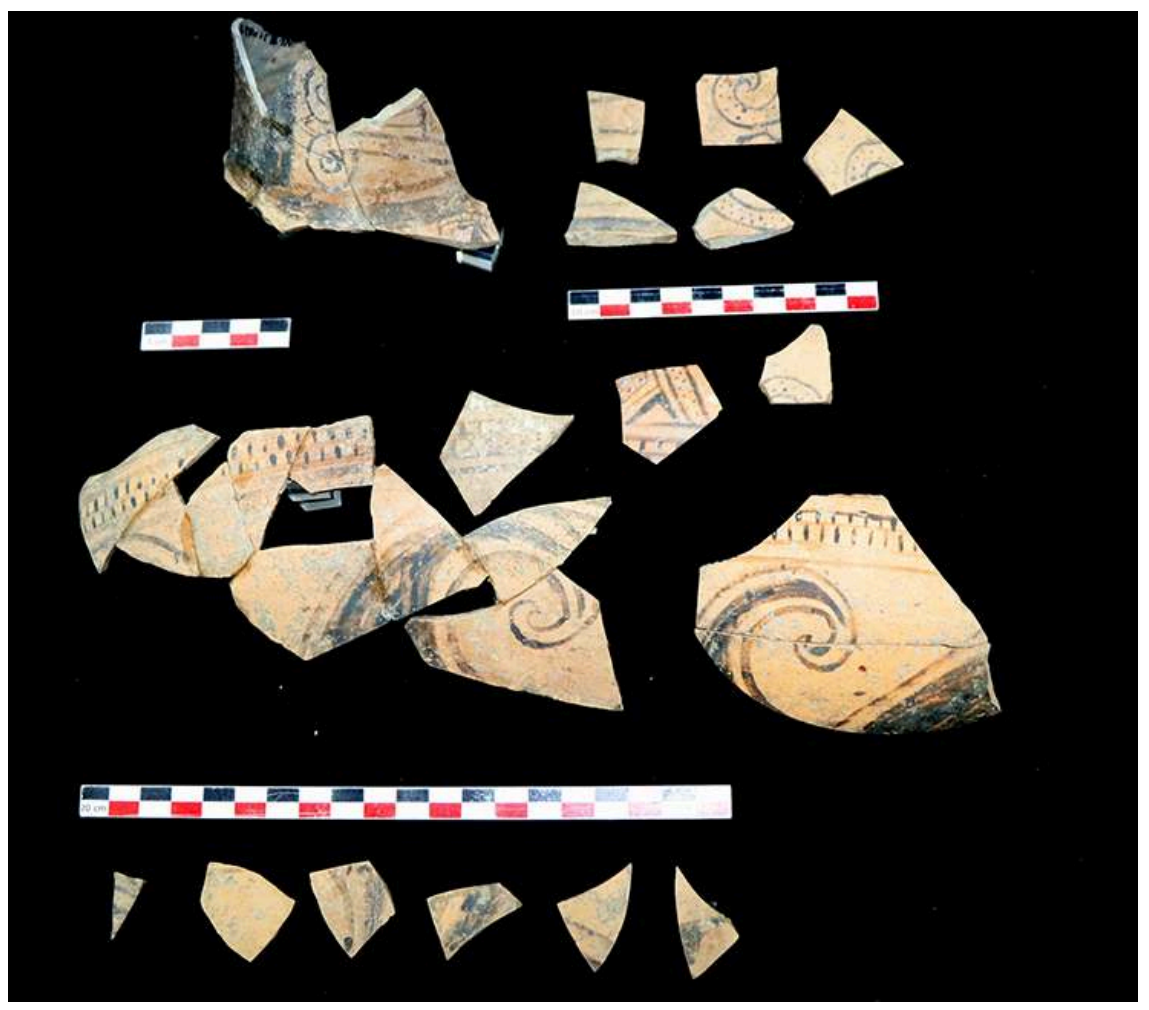

Mais il y a plus : de manière très significative, ce vase grec produit sur place, destiné à des pratiques libatoires et brisé après son usage rituel, a été disséminé dans différents contextes adjacents : non seulement nous le trouvons déposé sur les deux lits de galets que nous venons de voir (fig. 18, A1, A2), mais ses parois sont également présentes à l'intérieur de la fosse que nous avons fouillée en 2017 et 2018, qui se trouve à la limite nord-orientale de la grande structure avec sol et mur (fig. 18, A3 ; fig. 19 ; fig. 20, C ; voir infra). 
Fig. 18 - Détail de la planimétrie des secteurs Est, Nord et Ouest (DAO T. Ben Makhad).

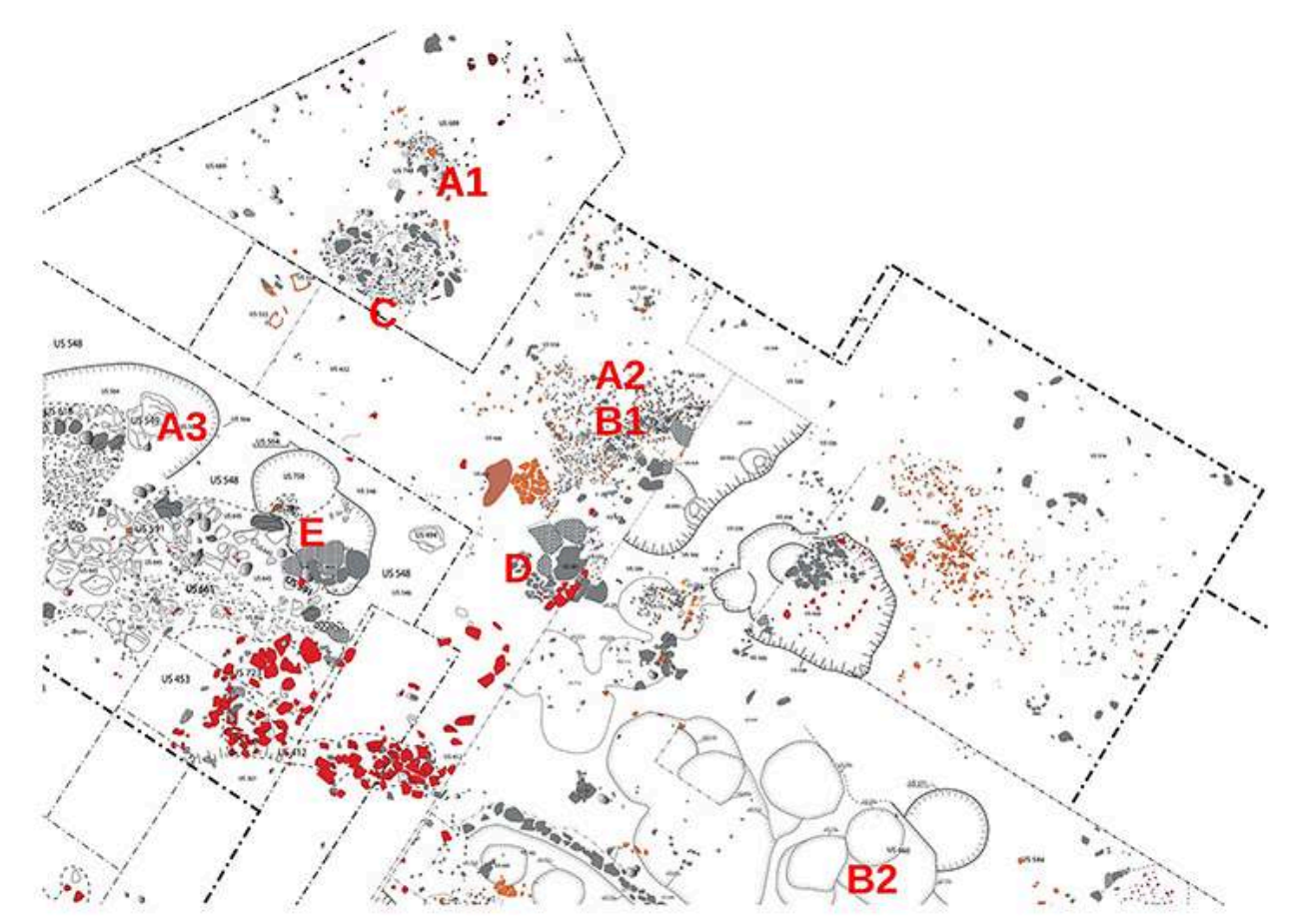

En définitive, la contemporanéité chronologique et l'homogénéité fonctionnelle de ces trois contextes peuvent être ultérieurement élargies, se prolongeant jusqu'à l'opération d'oblitération de la fosse cylindrique se trouvant plus à l'est, déjà décrite (fig. 5, L). En effet, à la triple correspondance dépositionnelle que nous venons de remarquer, nous devons ajouter une autre concordance fondamentale, elle aussi évoquée précédemment : la tête de serpent en terre cuite (fig. 11) retrouvée sur les bords de la fosse cylindrique (fig. 18, B2) et le segment de son corps provenant du lit de galets US 527 (fig. 18, B1). Ainsi, la dissémination des parties d'un autre objet, également doté d'un statut d'exception, dans les divers contextes rituels de cet espace cultuel de la colline (fig. 18, B1, B2) - répondant aux critères propres de la pratique rituelle de l' enchainment - permet de déduire la remarquable cohérence chronologique des actions rituelles qu'y avaient été accomplies.

\section{Le secteur Ouest : une structure qui s'agrandit progressivement}

L'aménagement rituel que nous venons de décrire se situe à quelques mètres au nord du grand bâtiment découvert l'année dernière à la limite nord-occidentale du plateau (fig. 1) ${ }^{13}$. Celui-ci est conservé au niveau des fondations - l'élévation ayant été démolie et a été recouvert, et protégé, par de la terre contenant des centaines de briques, toutes invariablement fragmentées intentionnellement, qui fort probablement appartenaient à son élévation. Les différents éléments de cette construction ont été installés à l'intérieur d'un grand creusement réalisé dans le terrain géologique. Elles comprennent un sol en fin cailloutis retrouvé en parfait état de conservation (fig. 19, US 640 ; fig. 20, A) et une grande structure de gros blocs de pierre, mélangés à de la terre en guise de ciment (fig. 19, US 591 ; fig. 20, B). 
Fig. 19 - Détail de la planimétrie du secteur Ouest (DAO T. Ben Makhad).

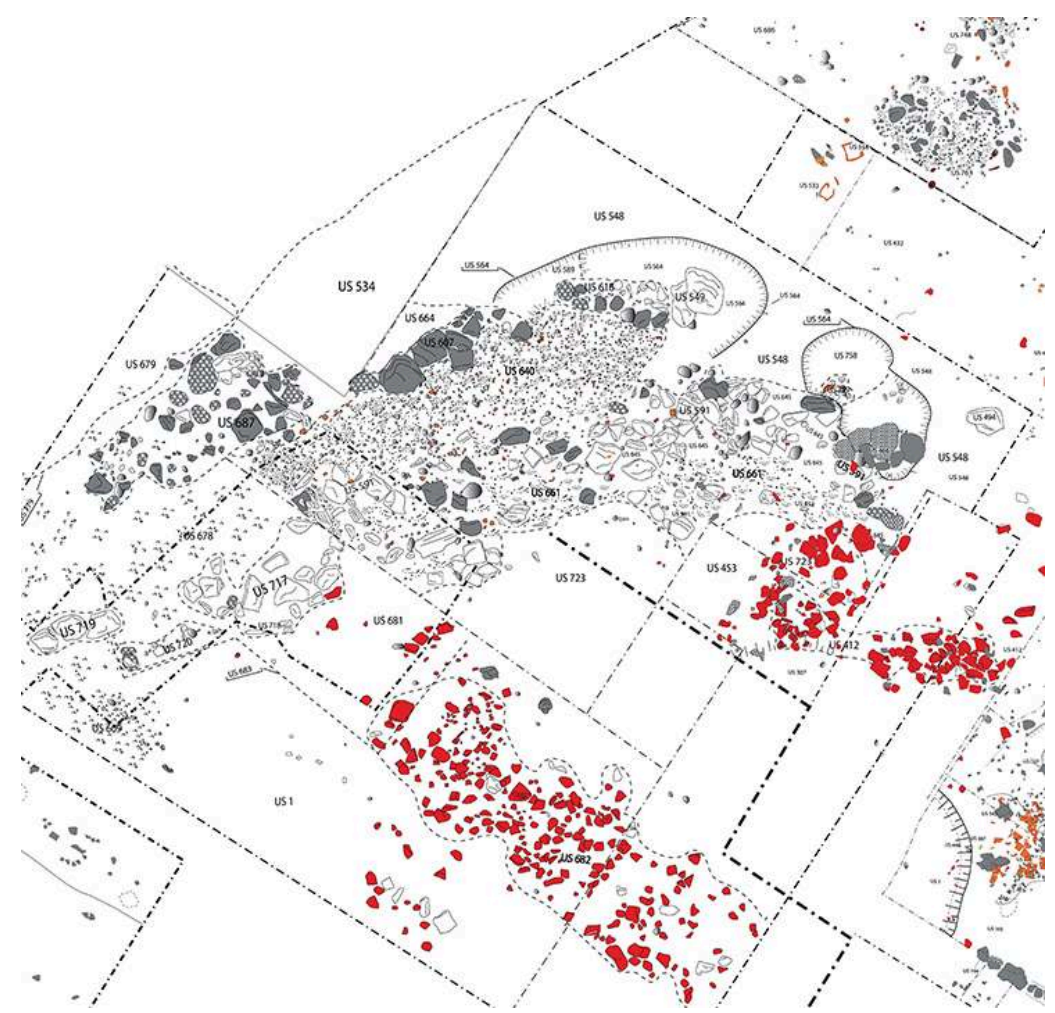

Fig. 20 - Photogrammétrie du secteur Ouest (élaboration T. Ben Makhad).

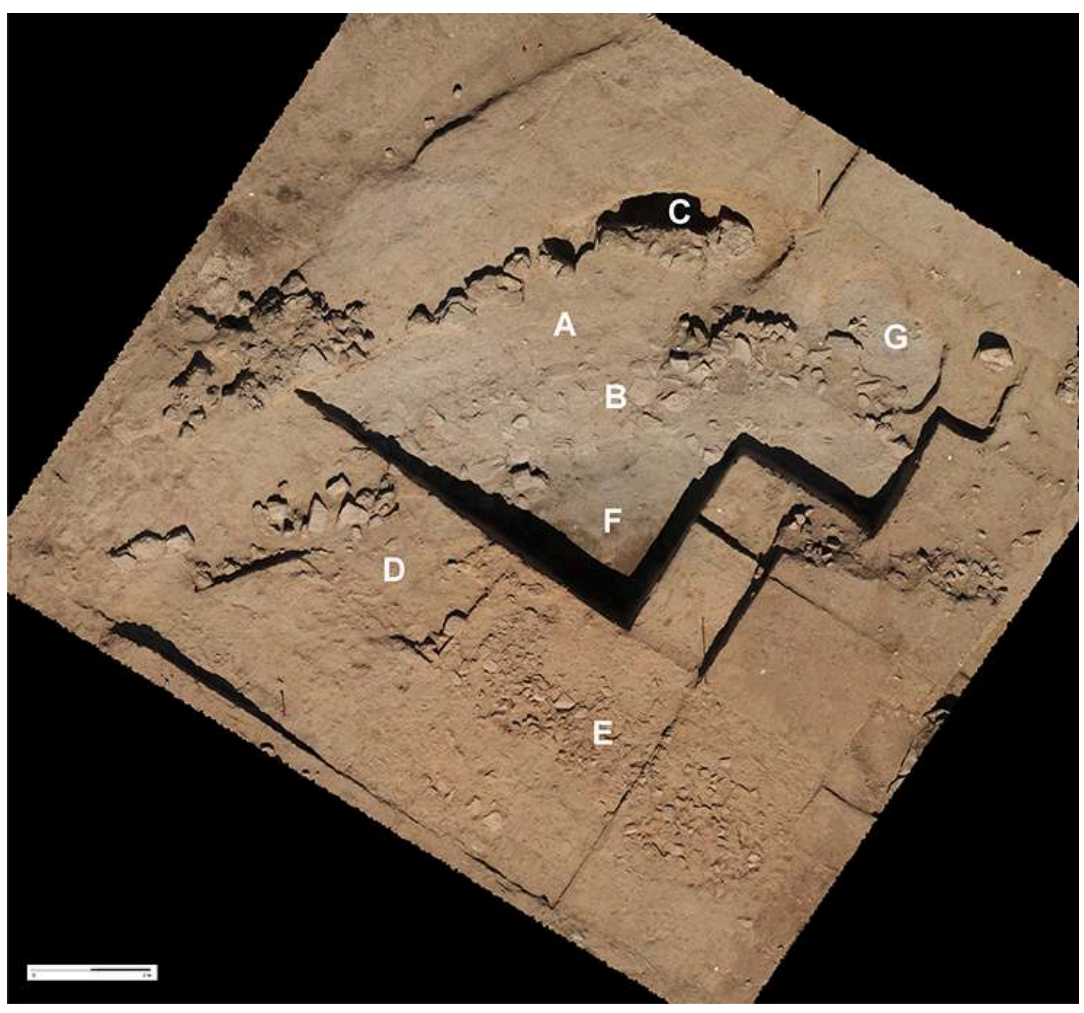

20 Nous rappelons que l'année dernière nous avons mis au jour, déposée sur ce sol, une petite sculpture en bronze, représentant la tête d'une image féminine de style 
dédalique, en cours de publication ${ }^{14}$. Il s'agit d'une découverte extrêmement importante, non seulement en raison de la valeur documentaire de l'objet, mais aussi parce qu'elle confirme la vocation cultuelle de ce bâtiment. Cette vocation avait d'ailleurs déjà été suggérée par la mise au jour d'une importante déposition rituelle réalisée à l'intérieur d'une fosse creusée au nord du sol (fig. 18, A3 ; fig. 19 ; fig. 20, C), caractérisée par un choix d'objets de prestige. À l'intérieur, parmi de nombreux os d'animaux, de la céramique achrome, monochrome et à impasto, des exemplaires de céramique proto-corinthienne d'importation, remarquables par leur quantité et leur qualité, dont les formes relèvent d'un contexte post-rituel à caractère libatoire et sacrificiel (des amphores, des skyphoi et des kotylai, un krateriskos, une œnochoé, une chytra, un louterion, des fragments de l'œnochoé de production locale discutée précédemment) ${ }^{15}$.

Nous avons cette année élargi la fouille en direction sud-ouest, dans l'objectif de mieux cerner l'orientation et la morphologie de ce bâtiment, sans toutefois avoir réussi encore à en comprendre la fonction. Un nouveau et très large segment de l'impressionnante couverture en briques a été dégagé (fig. 20, D ; fig. 21), permettant de se rendre ultérieurement compte de l'envergure des opérations d'oblitération ritualisée mises en place au moment de la démolition de ce bâtiment. Un secteur de cette compacte couverture, formant un espace rectangulaire de $3 \times 9 \mathrm{~m}$ environ, est caractérisé par une concentration majeure de briques, d'ailleurs parmi les mieux conservées de toute la colline (fig. 20, E; fig. 21). Son ampleur pose la question de la structure qu'elle était censée de protéger.

Fig. 21 - Secteur Ouest : la grande concentration de briques fragmentées, vue de l'Est, recouvrant les structures qui, en haut à droite de l'image, apparaissent partiellement mises au jour (cliché M. Denti).

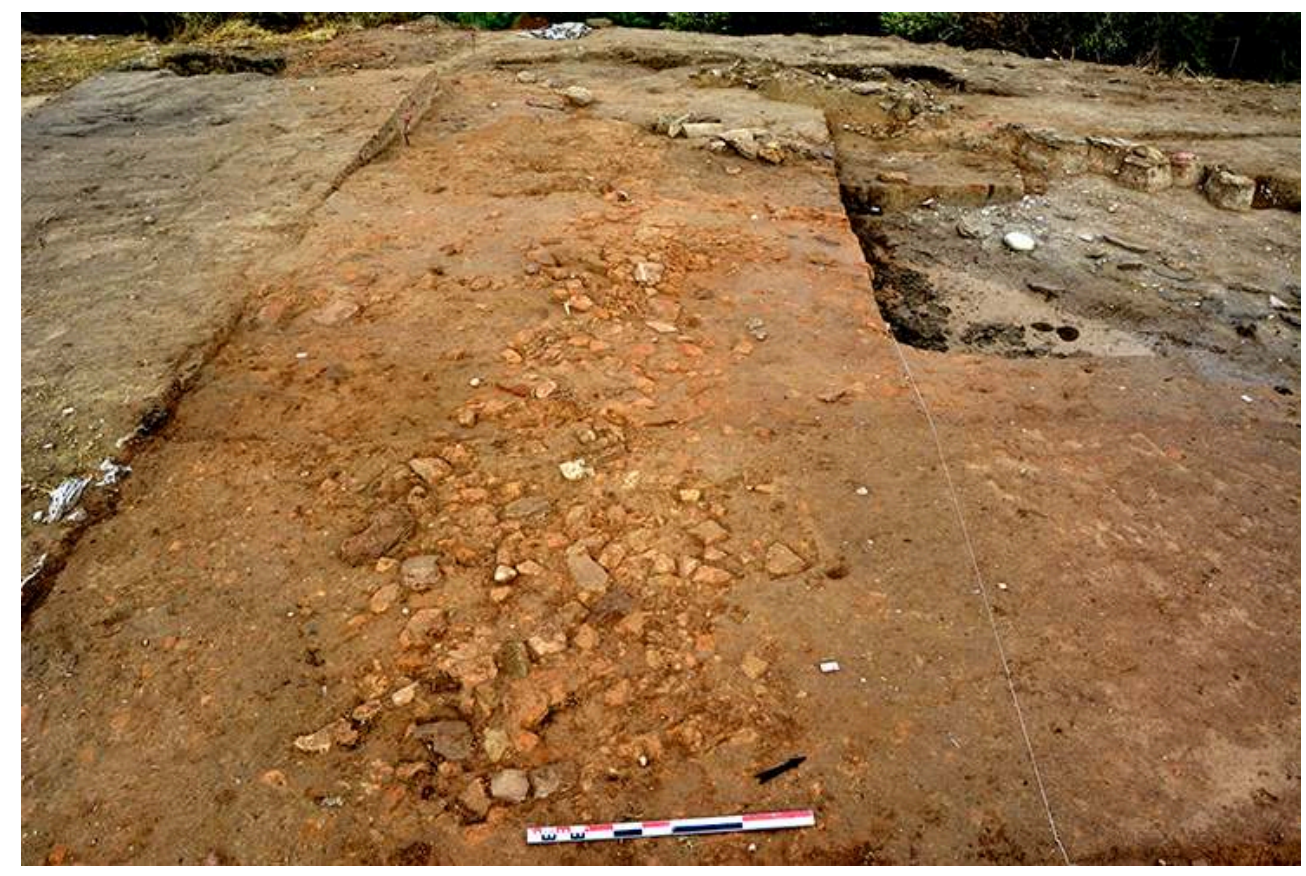

Un nouveau segment du pavement en cailloutis, dans lequel de minuscules fragments de céramiques et d'os émiettés ont été tassés pour en consolider la surface, a été également mis au jour en direction sud-ouest (fig. 19, US 640 ; fig. 20, A ; fig. 22). Tout au long de sa limite méridionale, l'imposante structure en pierres continue 
parallèlement dans la même direction du sol, en atteignant actuellement $10 \mathrm{~m}$ de longueur et 2 de largeur (fig. 19, US 591 ; fig. 20, B). Adossée au profil méridional de la structure, parmi les autres couches de terre grise-marron que nous avons commencé à enlever, il faut signaler notamment l'US 749, caractérisée par de la terre grise, mélangée à une grande quantité de charbon de bois, à des tessons de céramique noircie (monochromes et grands conteneurs), et à des grains carbonisés (fig. 20, F ; fig. 23). À l'extrémité nord-orientale de la structure en pierres nous avons intercepté deux fosses (ou peut-être une seule, bilobée) creusées dans le sol géologique de la colline (US 758 et 754, fig. 18, E; fig. 20, G). Dans la partie supérieure du remplissage de chacune, un exemplaire de fusaïole a été déposé. La terre de comblement, que nous n'avons pas encore enlevée, commençait à faire sortir de la céramique indigène, grecque locale et grecque d'importation.

Fig. 22 - Secteur Ouest : détail du sol en cailloutis US 640 (cliché M. Denti).

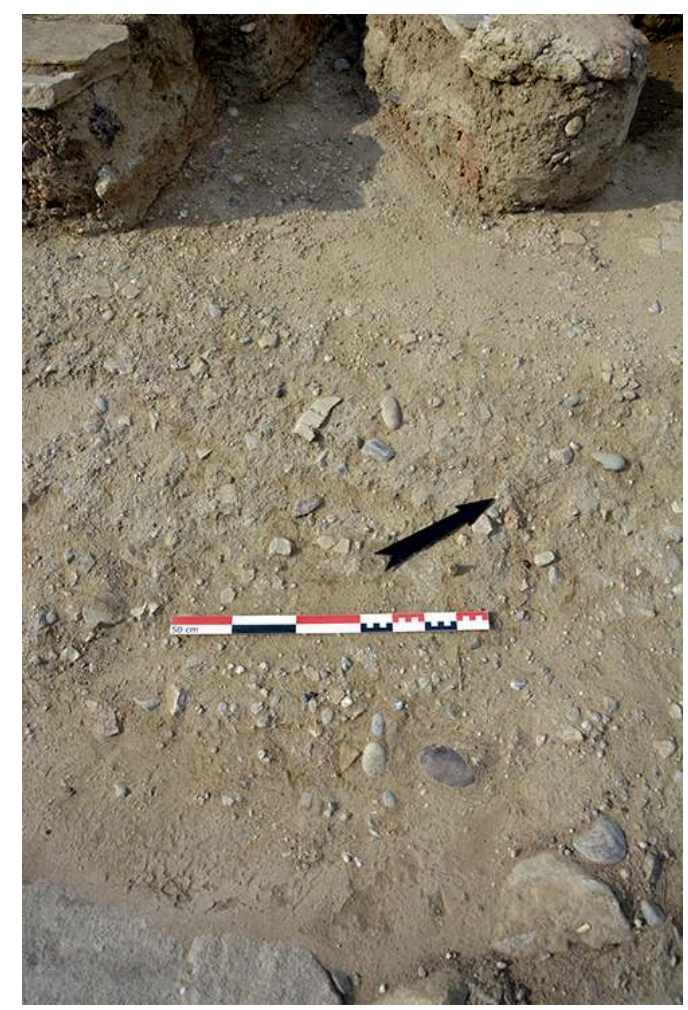


Fig. 23 - Secteur Ouest : les couches de terre longeant la limite méridionale de la structure en pierres, contentant du charbon de bois, de la céramique noircie et des grains (cliché M. Denti).

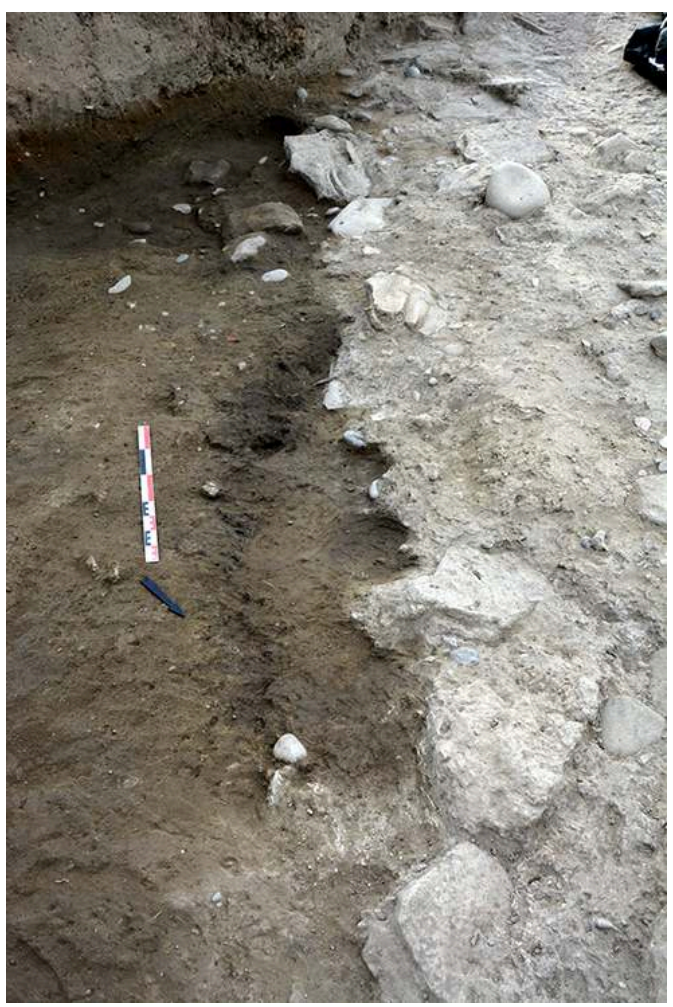

\section{BIBLIOGRAPHIE}

Chapman $2000=\mathrm{J}$. Chapman, Fragmentation in archaeology. People, places and broken objects in the Prehistory of South Eastern Europe, Londres-New York, 2000.

Denti 2013 = M. Denti, Incoronata. Les résultats de la dixième campagne de fouille (2012). Ruptures et continuités dans l'occupation du site entre VIIIe et VIIe siècle avant J.-C., dans Chronique des activités archéologiques de l'École française de Rome 2013.

Denti 2014 = M. Denti, Incoronata, la onzième campagne de fouille (2013). Les structures de l'âge du Fer, des composants de l'espace artisanal, un édifice absidé à vocation rituelle, dans Chroniques des activités archéologiques de l'École française de Rome, 2014.

Denti 2015 = M. Denti, Des biens de prestige grecs intentionnellement fragmentés dans un contexte indigène de la Méditerranée occidentale au VII siècle av. J.-C., dans K. Harrell, I. Driessen (dir.), THRASUMA. Contextualising the intentional destruction of objects in the Bronze Age Aegean and Cyprus, workshop in Louvain, novembre 2013, Louvain, 2015, p. 99-116.

Denti 2018a = M. Denti, Archilochos did not sail alone to the bountiful shores of Siris: Parian and Naxian potters in Southern Italy in the 7th century BC., dans D. Katsonopoulou (dir.), Paros IV. Paros and its colonies, fourth international conference on the archaeology of Paros and the Cyclades, Paros 11-14 June 2015, Athènes, 2018, p. 39-63. 
Denti $2018 \mathrm{~b}=\mathrm{M}$. Denti, Des pratiques rituelles perpétuées sur deux siècles d'occupation à Incoronata. La campagne de 2017, dans Chronique des activités archéologiques de l'École française de Rome, 2018.

Denti 2019 = M. Denti, Cultes et pratiques rituelles chtoniens à Incoronata. Campagne 2018, dans Chroniques des activités archéologiques de l'École française de Rome, 2019.

Denti 2020 = M. Denti, Di terra e di pietre, di legno e di ciottoli. Tecniche edilizie e funzioni architettoniche sulla costa ionica dell'Italia meridionale nell'età del Ferro, dans F. Pesando, G. Zuchtriegel (dir.), Abitare in Magna Grecia : l'età arcaica, Atti del Convegno di Napoli-Paestum, 15-16 mars 2018, Pise, 2020, p. 199-220.

Denti, sous presse a = M. Denti, De la mer Égée au golfe de Tarente. Une communauté grecque dans l'Ouest non grec au VII siècle avant J.-Chr., dans G. Tsetskhladze (dir.), International Conference Ionians in the East and West, Ampuries 26-29 octobre 2015, sous presse.

Denti, sous presse $\mathrm{b}=\mathrm{M}$. Denti, Ritual pebbles. Pebbles between the living and the dead in the Mediterranean Iron Age, dans Festschrift G. Tsetskhladze, sous presse.

Denti, sous presse $\mathrm{c}=\mathrm{M}$. Denti, Una piccola scultura dedalica in bronzo in un contesto cultuale indigeno dell'Italia meridionale, in Studi in memoria di E. Lippolis, sous presse.

Denti, en préparation = La céramique et les céramistes de Grèce de l'Est en Méditerranée occidentale. Les contextes archéologiques, les raisons de leur présence, dans A. Coulié (dir.), La céramique milésienne et apparentée à l'époque archaïque, Actes du colloque du Louvre, 27-28 novembre 2019, en préparation.

Kourou $2015=$ N. Kourou, Early Classical cemetery of Xobourgo at Tenos between tradition and modernity, dans H. Frielinghaus, J. Stroszeck, P. Valavanis (dir.), Griechische Nekropolen. Neue Forschungen und Funde, Möhnesee, 2019, p. 89-99.

\section{NOTES}

1. Toutes les dates sont avant J.-Chr.

2. Denti 2019.

3. Denti 2013 ; Denti 2014.

4. Denti 2019.

5. Denti 2019, fig. 13.

6. Chapman 2000.

7. Denti, sous presse a.

8. Kourou 2019, p. 95, pl. 22 ; Denti, sous presse b.

9. Denti 2018a; Denti 2020.

10. Denti 2018b ; Denti 2019.

11. Denti 2015, p. 110-111.

12. Des doutes subsistent sur la pertinence de tous les tessons à ce même exemplaire. Celui-ci est en cours d'étude par J. Mandić et moi-même. Il a été présenté lors d'un colloque à Paris en novembre 2019 : voir Denti en préparation.

13. Denti 2019.

14. Denti 2019, fig. 22, 23. Denti sous presse c.

15. Denti 2019, fig. 16-21. 
INDEX

Index géographique : Incoronata

Mots-clés : Incoronata, Âge du Fer, cultes chtoniens, espaces rituels, Grecs, Indigènes

\section{AUTEUR}

\section{MARIO DENTI}

Laboratoire LAHM, UMR 6566 CReAAH, Université Rennes 2 et IUF - mario.denti@wanadoo.fr 